\title{
Epidemiology and Outcomes of Takotsubo Syndrome in Hospitalizations With Systemic Sclerosis
}

\author{
Zainab Gandhi ${ }^{1}$, Hee Kong Fong ${ }^{2}$, Pritika Manaktala ${ }^{3}$, Faizan A. Malik ${ }^{4}$, Sejal Savani ${ }^{5}$, Neelesh Gupta ${ }^{6}$, \\ Rupak Desai $^{7}$ \\ 1. Internal Medicine, Geisinger Community Medical Center, Scranton, USA 2. Cardiovascular Medicine, University of \\ California Davis Medical Center, Sacramento, USA 3. Internal Medicine, Canton Medical Education Foundation, \\ Canton, USA 4. Internal Medicine, Texas Tech University Health Sciences Center at Permian Basin, Odessa, USA 5. \\ Public Health, New York University, New York, USA 6. Internal Medicine, Nazareth Hospital, Trinity Health Mid- \\ Atlantic, Philadelphia, USA 7. Cardiology, Atlanta Veterans Affairs Medical Center, Decatur, USA
}

Corresponding author: Rupak Desai, drrupakdesai@gmail.com

\section{Abstract \\ Background}

Systemic Sclerosis (SSc) is associated with chronic inflammation which leads to macrophage activation and thus vascular insult and fibrosis. Macrophage activation is shown to precede Takotsubo syndrome (TTS) which may be a common pathophysiologic link to SSc.

\section{Methods}

We queried the National Inpatient Sample (2008-2014) for adult SSc-related hospitalizations and TTS using relevant International Classification of Diseases Clinical Modification, 9th Revision codes. We assessed the prevalence and trends in TTS during this time. We further assessed demographics, comorbidities, and outcomes were in SSc with and without TTS. The primary outcomes of the analysis were all-cause mortality and in-hospital complications including cardiac arrest and acute myocardial infarction (AMI), arrhythmias, and venous thromboembolism, and stroke.

\section{Results}

A total of 213,728 SSc-related hospitalizations were found, of which 357 experienced TTS $(0.2 \%)$ with rising trends in TTS from $2008-2014$ ( $0.06 \%$ to $0.3 \%$, relative increase of $24 \%$, $\left.\mathrm{p}_{\text {trend }}<0.001\right)$. The TTS cohort was older (median age 68 vs 62 years), with $92.8 \%$ females and $80.1 \%$ white adults with TTS ( $\mathrm{p}<0.001)$. Comorbidities were higher in the TTS cohort including hypertension ( $62.2 \%$ vs. $51.5 \%, \mathrm{p}<0.001)$, dyslipidemia ( $41.5 \%$ vs. 22.8 , $\mathrm{p}<0.001)$, smoking $(28.9 \%$ vs. $20.1 \%$, $\mathrm{p}<0.001)$, peripheral vascular disease $(17.8 \%$ vs. $9.1 \%$, $\mathrm{p}<0.001)$, uncomplicated diabetes ( $18.1 \%$ vs. $11.9 \%, \mathrm{p}<0.001)$. The all-cause in-hospital mortality $(11 \%$ vs. 4.6\%; adjusted odds ratio $=1.82,95 \%$ confidence interval: $1.21-2.72$, $\mathrm{p}<0.005)$, cardiovascular complications like AMI ( $29 \%$ vs. $2.9 \%, \mathrm{p}<0.001)$, arrhythmias ( $38.9 \%$ vs. $21.5 \%$, p $<0.001$ ), and median length of stay [6 vs. 4

Received 08/11/2020 Review began $08 / 13 / 2020$ Review ended 08/15/2020 Published 08/21/2020

\section{(c) Copyright 2020}

Gandhi et al. This is an open access article distributed under the terms of the Creative Commons Attribution License CC-BY 4.0., which permits unrestricted use, distribution, and reproduction in any medium, provided the original author and source are credited. days] were significantly higher in the TTS cohort as compared to the non-TTS cohort.

\section{Conclusion}

This analysis revealed a nearly 10 times higher prevalence of TTS in SSc-related hospitalizations compared to the general inpatient population. Concomitant TTS occurrence in SSc-related hospitalizations led to nearly two times higher odds of all-cause mortality. Cardiovascular co-morbidities in SSc may increase the risk of TTS and worsened outcomes.

Categories: Cardiology, Internal Medicine, Rheumatology

Keywords: takotsubo syndrome, systemic sclerosis, outcomes, epidemiology, takotsubo cardiomyopathy, scleroderma, stress induced cardiomyopathy, apical ballooning syndrome, broken heart syndrome

\section{Introduction}

Systemic sclerosis (SSc) is a chronic autoimmune disease that is characterized by vascular endothelial dysfunction and fibrosis of the internal organs and skin. It is mainly associated with Raynaud phenomenon, pulmonary arterial hypertension, and scleroderma renal crisis [1]. The pathogenesis of SSc involves chronic inflammation, followed by vascular insult and thus fibrosis. Macrophage activation secondary to chronic inflammation can lead to the production of mixed pro-inflammatory and anti-inflammatory cytokines and chemokines such as interleukin-4, interleukin-3, tumor necrosis factor, interleukin-6, transforming growth factor-beta, and chemokine (C-C motif) ligand 2 (CCL2) which mediate the fibrosis in SSc [2]. Takotsubo syndrome (TTS) has shown to be preceded by prolonged macrophage inflammatory infiltration and proinflammatory cytokines [3]. Melchiorre et al. in 2008 reported the first case of TTS in SSc patients suspecting 
microvascular injury, myocardial fibrosis, and Raynaud phenomenon, which contributes to the development of TTS [4].

\section{Materials And Methods}

In this first large-scale analysis, we sought to understand the epidemiology and outcomes of TTS in patients admitted with SSc in the United States. We conducted a retrospective analysis on National Inpatient Sample (2008-2014) with the International Classification of Diseases Clinical Modification, 9th Revision codes of TTS (429.83) and SSc (710.1) to study the SSc-related admissions with vs without TTS.

The study population involved inpatient hospitalizations in the National Inpatient Sample from 2008 to 2014. We assessed the in-hospital outcomes of SSc-related admissions with TTS and without TTS using multiple study variables. Demographics like age, sex, and race were considered. Co-morbidities and inhospital outcomes associated with SSc-related admissions with TTS and without TTS were assessed.

The primary outcomes for the analysis were all-cause mortality and in-hospital complications including cardiac arrest and acute myocardial infarction (AMI), arrhythmias, and venous thromboembolism, and stroke.

Statistical Package for the Social Sciences (SPSS), version 24 (IBM Corp, Armonk, NY) was utilized to complete statistical analyses using defined strata/cluster designs. It was compared using the Chi-square test and Mann Whitney U test for categorical and continuous variables, respectively.

\section{Results}

The prevalence of TTS in SSc-related admissions was $0.2 \%(n=357)$ amongst the total SSc-related admissions $(n=213,728)$ from 2008 to 2014. The SSc-related admissions with TTS often showed elderly [68 (60-75) vs 62 (52-72) yrs; $\mathrm{p}<0.001$ ], white ( $80.1 \%$ vs $69.4 \%$; $\mathrm{p}<0.001)$, female $(90.8 \% \mathrm{v} / \mathrm{s} 80.1 \% \mathrm{p}<0.001)$ patients admitted for non-elective procedures $(87.2 \%$ vs $81.8 \%$; $\mathrm{p}=0.01$ ) as compared to the non-TTS cohort.

The prevalence of comorbidities including peripheral vascular disease (17.8\% vs $9.1 \%$; $\mathrm{p}<0.001$ ), hypertension $(62.2 \%$ vs $51.5 \%$; $<<0.001)$, smoking ( 28.95 vs $20.1 \%$; $p<0.001)$ and coagulopathy $(11.4 \%$ vs $6.1 \% ; \mathrm{p}<0.001)$ was higher in SSC-related admissions with TTS cohort vs without TTS. Higher prevalence of chronic pulmonary disease $(28.8 \%$ vs $22.2 \%$; $\mathrm{p}<0.001)$ and pulmonary circulation disorders $(21.1 \%$ vs $11.0 \%$; $\mathrm{p}=0.134$ ) was observed in SSc-related admissions with TTS vs without TTS.

Overall all-cause mortality in the SSc-TTS cohort was more than two times higher ( $11 \%$ vs $4.6 \%, \mathrm{p}<0.001)$ as compared non-TTS cohort. The SSc-TTS cohort had higher burden of in-hospital arrhythmias (38.9\% vs $21.5 \%$; $<<0.001)$, acute myocardial infarction ( $29 \%$ vs $2.9 \%$; $<<0.001)$, venous thromboembolism ( $6.9 \%$ vs $3.1 \%$; $<<0.001)$, stroke $(5.5 \%$ vs $1.7 \%$; $\mathrm{p}<0.001)$ and in-hospital cardiac arrest $(4.3 \%$ vs $1.7 \%$; $<<0.001)$ as compared to non-TTS cohort (Table 1 ).

\begin{tabular}{|c|c|c|c|c|c|c|}
\hline \multirow{2}{*}{ Variable } & & \multicolumn{2}{|c|}{ No Takotsubo Syndrome $(n=213,372)$} & \multicolumn{2}{|c|}{ lakotsubo synarome $(n=356)$} & \multirow{2}{*}{$\mathbf{P}$} \\
\hline & & Number & $\%$ & Number & $\%$ & \\
\hline \multirow[t]{4}{*}{ Age at admission } & Median [IQR] & $62[52-72$ & & $68[60-75$ & & $<0.001$ \\
\hline & $18-44$ & 27,441 & $12.9 \%$ & NA & NA & \multirow{3}{*}{$<0.001$} \\
\hline & $45-64$ & 90,129 & $42.2 \%$ & 138 & $38.7 \%$ & \\
\hline & $>=65$ & 95,801 & $44.9 \%$ & 219 & $61.3 \%$ & \\
\hline \multirow{4}{*}{ Sex } & Male & 33,863 & $15.9 \%$ & 26 & $7.2 \%$ & \multirow{3}{*}{$<0.001$} \\
\hline & Female & 179,493 & $84.1 \%$ & 331 & $92.8 \%$ & \\
\hline & White & 133,668 & $69.4 \%$ & 262 & $80.1 \%$ & \\
\hline & African American & 30,173 & $15.7 \%$ & 20 & $6.1 \%$ & \multirow{6}{*}{$<0.001$} \\
\hline \multirow{5}{*}{ Race } & Hispanic & 18,948 & $9.8 \%$ & 15 & $4.6 \%$ & \\
\hline & Asian or Pacific Islander & 3,419 & $1.8 \%$ & 15 & $4.7 \%$ & \\
\hline & Native American & 1,573 & $0.8 \%$ & NA & NA & \\
\hline & Others & 4,926 & $2.6 \%$ & 15 & $4.6 \%$ & \\
\hline & Non-elective & 174,215 & $81.8 \%$ & 311 & $87.2 \%$ & \\
\hline
\end{tabular}




\section{Cureus}

Type of admission

Elective

38,664

$18.2 \%$

46

$12.8 \%$

0.01

Comorbidities

Alcohol abuse

3,812

$1.8 \%$

NA

NA

0.582

Deficiency anemias

13,021

$6.1 \%$

$11.4 \%$

$<0.001$

Chronic pulmonary disease

60,887

$28.5 \%$

$34.4 \%$

0.013

$22.2 \%$

$28.8 \%$

0.002

Depression

47,345

$14.7 \%$

$16.5 \%$

0.328

Diabetes, uncomplicated

$11.9 \%$

$18.1 \%$

$<0.001$

Diabetes with chronic complications

25,327

$2.8 \%$

Drug abuse

6,078

$2.3 \%$

NA

0.001

4,875

Hypertension

109,961

$51.5 \%$

NA

0.514

Liver disease

9,672

$4.5 \%$

$62.2 \%$

$<0.001$

Obesity

12,831

$6.0 \%$

Peripheral vascular disorders

19,496

$9.1 \%$

Pulmonary circulation disorders

38,324

$18.0 \%$

Renal failure

33,362

$15.6 \%$

0.332

Solid tumor without metastasis

3,359

$1.6 \%$

Valvular heart disease

14,599

$6.8 \%$

48,727

$22.8 \%$

20,025

$9.4 \%$

42,914

$20.1 \%$

9,795

$4.6 \%$

$<0.001$

Arrhythmia

45,891

$21.5 \%$

$38.9 \%$

$<0.001$

Acute myocardial infarction

6,229

$2.9 \%$

$29.0 \%$

$<0.001$

Venous thromboembolic events

6,689

$3.1 \%$

$6.9 \%$

$<0.001$

Stroke

3,685

$1.7 \%$

$5.5 \%$

$<0.001$

In-hospital cardiac arrest

3,592

$1.7 \%$

20

$4.3 \%$

$<0.001$

Disposition

Routine
Transfer to short-term hospitals
Transfer to other (SNF, ICF)

121,770

$57.1 \%$

157

$44.0 \%$

6,096

$2.9 \%$

15

$<0.001$

34,140

$16.0 \%$

95

$4.2 \%$

$26.7 \%$

4 [2-7]

6 [3-12]

$<0.001$

27,952 [15085-53704]

62,560 [28956-115320]

$<0.001$

\section{TABLE 1: Systemic sclerosis-related admissions with versus without Takotsubo syndrome}

$\mathrm{P}<0.05$ indicates statistical significance. IQR, interquartile range; $\mathrm{MI}$, myocardial infarction; $\mathrm{PCI}$, percutaneous coronary intervention; CABG, coronary artery bypass grafting; SNF, skilled nursing facility; ICF, intermediate care facility. Note: Cell sizes less than 11 were indicated by NA as per the privacy guidelines under the Healthcare Cost and Utilization Project (https://www.hcup-us.ahrq.gov/db/publishing.jsp). 


\section{Discussion}

In our analysis, we found that SSc-related admissions with TTS were found to be $0.2 \%$, which is nearly 10 times higher than the prevalence of TTS $(0.02 \%)$ reported in the general inpatient population in the United States [5]. SSc-related admissions with TTS were seen more commonly in the elderly, white, and females, and patients with pre-existing comorbidities like hypertension, peripheral vascular disease, smoking, and coagulopathy. The observation of prevalence in the older female patients can be secondary to the postmenopausal state with decreased estrogen levels, as estrogen has shown benefit in coronary blood flow and preventing endothelial dysfunction [1]. The female predominance associated with both of these conditions could be the reason [1].

Endothelial dysfunction possibly contributed by smoking to the SSc-related TTS cohort. In addition, the pathophysiological process of SSc with increased endothelin and decreased nitric oxide and prostacyclin levels lead to increased pro-fibrotic factors and vascular fibrosis in the SSc-related TTS cohort. The compromise of pulmonary vascular endothelium in SSc, eventually leads to an increased prevalence of the comorbidity. There was a higher prevalence of in-hospital complications of venous thromboembolism and stroke in the TTS-SSc cohort. Increased coagulopathy due to platelet activation and enhanced coagulation disorders are also associated with SSC-related admissions with the TTS cohort $[1,6]$.

Furthermore, in SSc-related admissions with TTS, we found the all-cause mortality was double in comparison to the non-TTS cohort. Secondary outcomes like in-hospital arrhythmias, acute myocardial infarction, in-hospital cardiac arrest also showed a higher burden in the TTS cohort in comparison to the non-TTS cohort. Accelerated atherosclerosis due to increased immune response, and systemic inflammation leading to premature atherosclerosis is a widely known mechanism for SSc. Vasospasm in TTS contributes to exacerbating ischemia and myocardial fibrosis, which could be a possible explanation for the observed higher frequency of AMI in the TTS cohort. Arrhythmias are secondary to the conduction system and myocardial fibrosis, secondary to endothelial dysfunction in SSc [7]. Due to the higher burden of worse outcomes in the TTS cohort, it can lead to higher utilization of health care resources with increased length of stay as seen in our analysis. It can lead to higher utilization of financial, material and human resources in the healthcare system.

\section{Limitations}

Potential limitations of this study include billing and coding issues due to administrative data collection, lack of longitudinal follow-up, lack of data on the primary cause of death, lack of medication history and non-availability of laboratory results. Furthermore, there could be a possibility of residual confounders in the univariate analysis as the small sample of SSc hospitalizations did not allow us to explore comprehensive multivariable analysis. Despite these limitations, nationally representative cohorts of TTS and SSc enabled us to perform this analysis on one of the understudied subjects.

\section{Conclusions}

In conclusion, the prevalence of TTS in SSc-related admissions was $0.2 \%$. SSc-related admissions showed higher than two times the all-cause mortality, 10 times higher frequency of acute myocardial infarction and higher frequency of arrhythmias, venous thromboembolism, stroke, and acute cardiac arrest with statistical significance. This study highlights the possible relation of worse prognosis for SSc-related admissions with an episode of TTS versus without TTS.

\section{Additional Information \\ Disclosures}

Human subjects: All authors have confirmed that this study did not involve human participants or tissue. Animal subjects: All authors have confirmed that this study did not involve animal subjects or tissue. Conflicts of interest: In compliance with the ICMJE uniform disclosure form, all authors declare the following: Payment/services info: All authors have declared that no financial support was received from any organization for the submitted work. Financial relationships: All authors have declared that they have no financial relationships at present or within the previous three years with any organizations that might have an interest in the submitted work. Other relationships: All authors have declared that there are no other relationships or activities that could appear to have influenced the submitted work.

\section{References}

1. Patel H, Aggarwal NT, Rao A, et al.: Microvascular disease and small-vessel disease: the nexus of multiple diseases of women. J Womens Health. 2020, 29:770-779. 10.1089/jwh.2019.7826

2. Bhandari R, Ball MS, Martyanov V, et al.: Profibrotic activation of human macrophages in systemic sclerosis . Arthritis Rheumatol. 2020, 72:1160-1169. 10.1002/art.41243

3. Scally C, Abbas H, Ahearn T, et al.: Myocardial and systemic inflammation in acute stress-induced (takotsubo) cardiomyopathy. Circulation. 2019, 139:1581-1592. 10.1161/CIRCULATIONAHA.118.037975

4. Melchiorre D, Bernardo P, Conforti ML, et al.: Tako-tsubo-like syndrome in systemic sclerosis: a sign of myocardial Raynaud phenomenon?. Ann Rheum Dis. 2008, 67:898-899. 10.1136/ard.2007.069500 


\section{Cureus}

5. Deshmukh A, Kumar G, Pant S, Rihal C, Murugiah K, Mehta JL: Prevalence of takotsubo cardiomyopathy in the United States. Am Heart J. 2012, 164:66-71. 10.1016/j.ahj.2012.03.020

6. Khera R, Light-McGroary K, Zahr F, Horwitz PA, Girotra, S: Trends in hospitalization for takotsubo cardiomyopathy in the United States. Am Heart J. 2016, 172:53-63. 10.1016/j.ahj.2015.10.022

7. Lambova S: Cardiac manifestations in systemic sclerosis . World J Cardiol. 2014, 6:993-1005. 10.4330/wic.v6.i9.993 